Cahiers d'études africaines

\title{
Héritages et réparations en quête d'une justice pour le passé ou le présent
}

Bogumil Jewsiewicki

\section{(2) OpenEdition}

1 Journals

Édition électronique

URL : http://journals.openedition.org/etudesafricaines/4514

DOI : 10.4000/etudesafricaines. 4514

ISSN : $1777-5353$

Éditeur

Éditions de l'EHESS

Édition imprimée

Date de publication : 1 janvier 2004

Pagination : 7-24

ISBN : 978-2-7132-1823-1

ISSN : 0008-0055

\section{Référence électronique}

Bogumil Jewsiewicki, « Héritages et réparations en quête d'une justice pour le passé ou le présent », Cahiers d'études africaines [En ligne], 173-174 | 2004, mis en ligne le 22 novembre 2013, consulté le 30 avril 2019. URL : http://journals.openedition.org/etudesafricaines/4514 ; DOI : 10.4000/ etudesafricaines.4514 


\section{Bogumil Jewsiewicki}

\section{Héritages et réparations en quête d'une justice pour le passé ou le présent*}

D'un point de vue chronologique, le recours à l'argumentation construite à partir de la notion de réparation a succédé dans les rapports Nord-Sud à l'omniprésence de la notion de développement. Dans le domaine des relations entre les nations et les communautés, développement et réparation renvoient aux mêmes notions de base. À l'articulation entre le passé et le futur, le présent ${ }^{1}$ est saisi comme un moment pour redresser des injustices héritées du passé. Qu'il s'agisse de développement, auquel certains pays contribuent par des capitaux et par l'expertise et d'autres pays par des ressources et de la main-d'œuvre, ou de réparations à l'égard des injustices commises dans le passé, mais dont les effets perdurent, l'idée maîtresse est de rétablir l'état des choses tel qu'il aurait dû advenir en l'absence des

* Plusieurs articles ont été présentés lors des rencontres que j'ai initiées. Il s'agit du colloque de février 2004, organisé par l'Agence universitaire de la Francophonie, l'Université de Maurice et la Chaire de recherche du Canada en histoire comparée de la mémoire pour Françoise Vergès et Jocelyn Chan Low. Jean OMASOMBo et Gauthier DE VILLERS ont présenté leurs textes lors de la semaine Mémoires de Lubumbashi, en août 2002, organisée par cette chaire et l'Université de Lubumbashi. Le texte de Bennetta Jules-Rosette \& David Coplan ainsi que le dossier Burundi ont été présentés lors des sessions organisées en 2002 avec Bob WHITE à l'African Studies Association.

La rencontre qui clôture le cycle marquera le lancement du numéro. Les contributions de l'Institut français en Afrique du Sud (IFAS) et de l'École des hautes études en sciences sociales rendent possible la participation de Rehana VALLY et offrent l'occasion de souligner le $10^{\mathrm{e}}$ anniversaire des premières élections démocratiques en Afrique du Sud et de témoigner de la vigueur de la collaboration avec l'IFAS, avec l'University of Witwatersrand et le Center for the Study of Violence and Reconciliation de Johannesburg. La section Histoire du Temps présent du Musée royal de l'Afrique centrale et la Chaire de recherche du Canada en histoire comparée contribuent également à sa tenue.

Qu'il me soit permis de remercier Marie-José Jolivet et Roger Botte pour leur aide.

1. Dans le premier cas, il n'est que transition vers le futur, dans le second, il est un espace temporel qui absorbe le futur et le passé qu'il «présentise » (HARTOG 2003). 
facteurs justifiant la réparation ou l'aide au développement. Une certaine utopie tournée vers le futur distingue la première option du «présentisme » de la seconde qui privilégie la « réparation » au présent des injustices passées. La légitimation d'un appel à l'action en faveur des sociétés et des personnes «lésées » met en avant l'argument selon lequel un monde plus juste et plus équitable, qu'il le soit au futur ou au présent, sera meilleur pour tous. Pour le Nord, la privation temporaire d'une fraction des dépenses publiques ou privées serait récompensée par une vie meilleure et surtout par la satisfaction morale d'avoir corrigé une injustice.

Le sens commun de la culture de globalisation de tradition occidentale, aussi laïcisée soit-elle, qui admet la possibilité de rétablir l'état antérieur par l'aveu et la repentance, puise dans la tradition de l'antifascisme occidental et dans l'enseignement catholique laïcisé portant sur la dette envers Dieu pour les péchés des hommes. Le déplacement d'accent de l'économie (identifiant les torts et les remèdes aux transferts des ressources) au politique et à l'éthique a mis en avant la notion de réparation. Moins apparents, les principes du libéralisme n'en sont pas pourtant absents. Anthony Appiah remarque dans ce numéro que la notion d'héritage d'un bien dont la possession autorise l'individu à exercer dans la durée une certaine maîtrise de son identité et de son existence, nourrit le discours sur les réparations. Sans prétendre restaurer à l'identique le passé, les réparations visent à replacer la victime ou ses descendants - personnes ou communautés — là où elles auraient dû se trouver si elles avaient conservé le contrôle de leurs biens et de leur cadre de vie matériel.

Étymologiquement, rappelle Appiah, réparer c'est restaurer ou soulager la victime d'un tort d'au moins trois manières : en restituant les biens ou le cadre de vie (économique, politique, etc.) ; en reconnaissant la responsabilité du tort commis; en rétablissant la relation pervertie par le tort subi. L'acte de réparation serait matériel ou expressif, par exemple une demande publique de pardon. Ce dernier est cependant rarement accepté comme étant suffisant, surtout si le tort fait aux victimes avait apporté à ses auteurs des avantages matériels ou politiques, ce qui est le cas de l'esclavage des temps modernes. Les relations entre l'Occident industrialisé et l'Afrique noire, ou encore entre les communautés nationales d'ascendance «blanche » et « noire » aux États-Unis, au Brésil, aux Antilles ou dans l'océan Indien sont marquées du sceau du « péché » originel, de la déshumanisation et de l'appropriation d'une personne et de sa force de travail, par une autre. Sa nature criminelle a été reconnue sans réserve par la loi française, proposée par Christiane Taubira-Delannon, deputée de la Guyanne, votée à l'unanimité par l'Assemblée nationale française le 6 avril 2000, et promulguée le 21 mai 2001; des regrets ont été exprimés et une reconnaissance moins nette de la traite des esclaves comme crime contre l'humanité est intervenue à Durban en septembre $2001^{2}$.

2. Michel Giraud présente dans l'article qui fait partie de ce numéro les débats qui ont eu lieu à l'Assemblé nationale. 
Dans les parties du monde citées, développées grâce au travail des esclaves noirs, les économies et les sociétés auraient sans doute été très différentes sans leur apport. Cependant, remarque Appiah, le recours au travail servile n'était pas un facteur uniformément favorable pour tous les Blancs ; sa concurrence aurait pu faire baisser les salaires de certains travailleurs blancs. Par ailleurs, certains esclaves affranchis sont devenus, à leur tour, propriétaires d'esclaves ${ }^{3}$. Si, à la place des individus, la communauté nationale noire bénéficiait de réparations, celles-ci, prélevées aujourd'hui sur des fonds publics, auraient pour effet que de nombreux contribuables noirs se paieraient des réparations à eux-mêmes. Longue est la liste des difficultés que soulèvent les réparations matérielles pour des actes qui remontent à deux siècles, voire plus. La tâche est compliquée davantage par la participation à la traite des esclaves de nombreux États africains indépendants de l'époque. «Toute recherche de responsabilité collective risque de se perdre dans une relecture globale de l'histoire humaine tout entière depuis au moins le $\mathrm{XV}^{\mathrm{e}}$ siècle », conclut le Rapport Debray ${ }^{4}$.

Quant à la colonisation, tous les États contemporains d'Afrique noire, à l'exception peut-être de l'Éthiopie, sont des produits de l'empire et n'auraient donc pas existé dans leur identité actuelle, dans leurs frontières actuelles en absence de la colonisation. À quel titre compenser les institutions et ces nations façonnées par des États, se demande Appiah ? Il existe évidemment des individus dont certains sont encore en vie, envers lesquels des administrations coloniales ont sans doute contracté des obligations dont elles ne se sont pas acquittées, mais les États africains ont légalement hérité de ces obligations. Appiah et Giraud concluent contre les réparations, dont l'argument tourné vers le passé constitue selon eux, le point le plus faible, le vice caché, en quelque sorte, de l'exigence de justice et d'équité dans le monde. Vouloir corriger les inégalités et les injustices actuelles ne gagne rien du rappel des torts commis jadis. Nous faisons partie du présent et en avons la responsabilité, nous devons donc agir au présent en honorant nos propres principes et nos engagements. La généalogie du mal ne porte en

3. Il est utile de renvoyer le lecteur au livre d'Ira BERLIN (2004) qui analyse l'évolution en Amérique, pas du tout linéaire, de la compétition entre diverses formes de travail et divers groupes de travailleurs tant blancs que noirs. Des esclaves ont également été propriétaires d'esclaves en Afrique, voir Roger BotTe (2001).

4. François Blancpain \& Marcel Dorigny, Réparations pour l'esclavage?, «Rapport au ministre des Affaires étrangères, Dominique de Villepin, du Comité indépendant de réflexion et de propositions sur les relations franco-haïtiennes », janvier 2004, annexe I, p. 80. Ce texte sera subséquemment cité comme Rapport Debray. Je remercie Carlo Célius de me l'avoir communiqué. Même la mémoire de l'ascendance esclave comme fondement de l'identité n'est pas sans poser de sérieux problèmes à ceux qui pourraient s'en réclamer comme le souligne, dans ce numéro, Gérard BARTHÉLÉMY : «On ne peut s'empêcher de penser que l'ancien esclave ne se prévaut de ce statut et de son émancipation que quand il s'agit d'essayer d'agir, par sa mémoire, sur l'ancien maître et éventuellement sur sa culpabilité. » 
elle ni la recette ni même la raison d'agir pour l'extirper, le présent devrait s'en charger autant pour définir l'action que pour la légitimer. Il y a dans cette position une rupture radicale avec l'idée de dette et, dans la logique de son argument, avec le fondement tant chrétien que libéral du poids et de l'importance de l'héritage pour la préservation de l'identité, de l'autonomie et de la justice dans le monde ${ }^{5}$.

Étant historien plutôt que philosophe, je ne reviendrai que sur la question de la dette et de l'héritage comme nerf de la guerre de l'historicité, censée être la condition indispensable à l'identité et à l'autonomie du sujet moderne, qu'il s'agisse d'une personne ou d'une collectivité — une nation par exemple. Il faut cependant rappeler très brièvement l'argument classique en faveur des réparations. Ses auteurs convoquent en premier lieu les obligations contractées dans le passé. Elles constituent, selon eux, une dette à l'égard de Dieu, de l'humanité que les Lumières ont placée à la place de Dieu. Le devoir de réparer les péchés des ancêtres découle du fait que, même malgré nous, nous en sommes les héritiers.

Alors qu'Appiah se place dans la perspective d'un présent responsable de son propre devenir, Ajayi, dont le texte rassemble et condense les principaux arguments des avocats des réparations qui seraient dues par l'Occident à l'Afrique, conceptualise le présent comme découlant du passé. Le lecteur pourrait être surpris de lire dans ce numéro un plaidoyer plutôt qu'une analyse de sciences sociales à laquelle il s'attendrait de la part d'un éminent historien. Il faut noter qu'Ajayi a prononcé sa conférence en sa qualité de l'un des derniers membres d'un groupe de pression dont Rhoda HowardHassmann décrit les activités. Son article situe très bien le contexte politique de l'appel aux réparations, lancé au nom de l'Organisation de l'unité africaine (OUA). Il en dresse, pour le lecteur, un bref historique de l'économie politique. La formulation et la promotion de ces revendications sont suivies, dans le cadre de la politique nigériane et dans celui des dernières années d'existence de l'ouA. Peu connu en Europe, en particulier par les francophones, le procès que les promoteurs des réparations intentent à l'Occident connaît une audience plus attentive dans les milieux radicaux africainsaméricains aux Etats-Unis (Robinson 1999). Le réquisitoire d'Ade Ajayi a été présenté en décembre 2002, au colloque annuel de l'African Studies Association, au titre de Distinguished Abiola Lecture. Il faut noter que le

5. L'égalisation des chances de promotion sociale, la lutte contre la discrimination raciale, qui est sans aucun doute un héritage de la traite des esclaves et de la colonisation, nécessitent une action au présent le plus immédiat qui n'a pas besoin de s'appuyer sur l'évidence des crimes du passé. Cette réparation symbolique, dont l'importance est capitale, ne constitue cependant que par abus de langage une réparation, insiste dans ce numéro Michel GiRAud. Pourtant, elle semble actuellement la forme de réparation la plus répandue, même si elle n'aboutit dans la majorité des cas qu'à dire une "vérité » historique et à lui reconnaître sa place dans l'espace public. Voir aussi à ce sujet Serge MAM LAM FoucK (2001) et Nassirou BAKO ARIFARI (2001) qui s'interroge sur la place de la mémoire de la traite des esclaves dans les formulations des identités africaines. 
richissime homme d'affaires et président élu du Nigeria, Bashorun M. K. O. Abiola, qui n'a jamais pu assumer cette fonction, et à la mémoire duquel la conférence a été créée, a soutenu politiquement et financièrement le mouvement des réparations. Comme le signale Rhoda Howard-Hassmann, depuis sa mort et depuis la reforme de l'ous suivie de sa transformation en Union africaine, le mouvement est en perte de vitesse. Prendre connaissance du document que constitue cette Distinguished Abiola Lecture permet de pleinement apprécier, en particulier dans le contexte politique du multiculturalisme nord-américain, l'analyse critique que présentent Anthony Appiah et Michel Giraud du mouvement en faveur des réparations.

Le Groupe de personnalités éminentes, un groupe de pression chargé par l'ouA de promouvoir la cause des réparations, s'appuie sur l'évidence de l'exploitation des Africains aux époques de la traite des esclaves et de la colonisation, événements de longue durée responsables des drames humains en Afrique et dans la diaspora. Il y aurait ainsi, argumente Ade Ajayi - l'un des trois derniers membres actifs du groupe —, l'obligation morale et politique d'une "Réparation ». La traite des esclaves des temps modernes et la colonisation sont, au moins partiellement, reconnues comme crimes contre $1^{\prime}$ humanité, donc imprescriptibles ${ }^{6}$. Il ne reste qu'à en tirer les conclusions politiques et éthiques selon le modèle des réparations pour l'extermination des Juifs d'Europe. S'y soustrayant, l'Occident se rendrait une fois de plus coupable de discrimination raciale ${ }^{7}$.

Dans le cadre des réparations dont bénéficie l'État d'Israël, les survivants et les descendants des victimes sont convoqués à titre d'expérience historique à laquelle se réfèrent toutes les demandes récentes de réparation. Cependant, les situations concernées par ces demandes et leurs contextes historiques diffèrent considérablement, parfois radicalement, de l'expérience saisie comme modèle. Aussi important qu'il soit d'analyser la légitimité et l'efficacité politique de cet alignement, ce n'est pas le but de ce numéro d'en analyser les raisons. Notre intention est de rassembler les analyses qui examinent les demandes et les discours de réparation et de restitution soit à l'Afrique comme entité politique, soit aux institutions nationales, soit aux Africains (y compris ceux qui vivent aujourd'hui dans les diasporas) et à leurs descendants, pour les torts causés par la traite des esclaves à l'époque moderne opérée par et pour le compte des acteurs occidentaux, la colonisation et la discrimination raciale à l'encontre des individus d'origine africaine

6. L'imprescriptibilité concerne la responsabilité pénale des auteurs des crimes en tant qu'individus, mais pas leurs descendants ce qui impliquerait la responsabilité collective.

7. Michel GiRAUD rappelle, dans ce numéro, que lors des débats à l'Assemblée nationale française, qui ont précédé l'adoption de la loi déclarant la traite des esclaves crime contre l'humanité, de part et d'autre du spectre politique français, il était question d'annulation de la dette, d'aide au développement de l'Afrique, d'un Plan Marshall pour les anciennes colonies, etc. Lors des manifestations publiques aux Antilles, l'exigence d'une réparation matérielle a été maintes fois posée, comme ce fut le cas à Durban en 2001. 
et de peau noire ${ }^{8}$. Le continent africain et les territoires de ses diasporas (résultats de la traite des esclaves et de la colonisation) en constituent l'aire géographique. Par la nature même des demandes et des offres de réparations, de restitutions et de réconciliations (pardon), le dossier porte sur le présent, même si la justification de la majorité des demandes nous ramène sans cesse vers le passé. Le débat actuel est marqué par l'urgence de l'action à entreprendre afin de réparer les torts, de restituer aux victimes ou à leurs descendants ce dont ils ont été spoliés, de rétablir la justice et l'égalité. Les torts causés jadis seraient la source d'une dynamique qui creuse continuellement l'écart entre le monde occidental, héritier politique et bénéficiaire des auteurs des crimes ${ }^{9}$, et les victimes passées et présentes. Redresser et réparer le passé serait le meilleur, voire le seul, moyen de garantir un avenir plus juste et plus équitable. Dans ce précepte, le présent ne serait qu'une articulation, un espace et un temps de passage sans dynamique propre.

L'article d'Achille Mbembe a le grand mérite de défendre le point de vue opposé, d'attirer l'attention sur l'autonomie de l'évolution politique et sociale des sociétés africaines contemporaines. Il s'inscrit en faux contre ceux qui, faisant fi du contemporain, pensent et agissent comme si l'Afrique indépendante était aussi immobile qu'aurait été l'Afrique précoloniale de l'anthropologie de jadis, ceux qui emprisonnent le présent dans la fatalité de l'héritage du passé. Sans aucun doute, contrairement à leur intention, les habitants du continent, dont le présent serait mis au monde par la traite et la colonisation, paraissent incapables d'assumer la responsabilité de leur présent autrement qu'en se tournant vers d'anciens maîtres que ce soit pour l'assistance ou pour les réparations. Sans disculper, pour autant, l'Occident

8. Aussi oiseuse qu'est cette longue énumération, elle est indispensable ici, compte tenu de l'exclusion de la traite dite arabe, du réquisitoire du Groupe des personnalités éminentes. Ali Mazrui, politologue américain d'origine kenyane, la justifie par l'absence de discrimination raciale dans la traite opérée par des agents de religion musulmane. Alors qu'en Occident moderne (incluant les colonies américaines et les îles de l'océan Indien) il n'y avait d'esclaves que les Noirs, dans le monde musulman un individu de n'importe quelle origine pouvait devenir esclave. Peu importe les mérites de la preuve de la thèse de Mazrui, que j'estime difficile à soutenir, il est pour nous important de retenir l'amalgame entre esclave et Noir qui, évidemment, heurterait tout autant les descendants des marrons que tous ceux qui justement se réclament de leur mémoire et dressent des monuments en leur mémoire en Haïti, à l'île Maurice et ailleurs. Apparemment les premiers esclaves déportés aux États-Unis n'étaient pas des Africains noirs mais des Métis (Créoles). Pourtant George FrEDRICKSON (2004 : 34), un des meilleurs spécialistes états-uniens de l'esclavage, vient d'écrire ; « Neither Koran nor Islamic law gave any sanction to racism, and Muslims always held white as well as black slaves. But in practice there was a tendency, which may have influenced southern Europeans who came in contact with Islamic slavery in the fourteenth and fifteenth centuries, to discriminate among slaves on the basis of colour. »

9. Michel GiRAud considère, dans ce numéro, qu'il est impossible de soutenir que les peuples des anciennes puissances coloniales ont, dans leur ensemble, bénéficié et bénéficient encore des richesses accumulées du fait de la traite et de l'esclavage. 
de ses responsabilités qui se situent dans le présent, il est indispensable de le «provincialiser », surtout à l'égard du présent et de l'avenir des peuples noirs, comme le font de plus en plus les intellectuels indiens à l'égard de l'empire britannique ${ }^{10}$.

La démarche de Mbembe est indispensable pour quiconque s'interroge sur les moyens de réformer de l'intérieur - ce qui n'exclut pas une coopération externe - la situation dramatique que connaissent de nombreuses régions d'Afrique et certaines de leurs diasporas. Le débat sur les responsabilités historiques de l'Occident et sur d'éventuelles réparations ne devrait pas en faire abstraction au risque de s'engager dans le cul-de-sac d'une nouvelle imposition autoritaire qui, étant bien au fait du passé, serait peu instruite du présent. Mbembe observe que, dans l'Afrique d'aujourd'hui, on accorde « une place centrale à la pensée et à la pratique du pouvoir comme pensée et pratique de la guerre », activité de « destruction de laquelle découle une relative jouissance » de consommer/détruire les richesses naturelles et de donner/recevoir la mort. On peut y voir les contractions d'un difficile accouchement de la maîtrise locale de la marchandisation (commodification). Il s'agit d'une naissance dangereuse, mais il est impossible d'en faire l'économie en pensant pourvoir l'empêcher d'advenir soit par un retour au passé, soit en exorcisant l'impact de l'Occident.

À l'exception des bibliothèques religieuses — islamique ou chrétienne d'autres bibliothèques que les Africains avaient en commun, coloniales ou anti-coloniales, sont désactualisées, et la gouvernementalité par la guerre cherche à se légitimer sur des débris de savoirs et des fragments de discours disparates. Les significations des imaginaires sociaux se trouvent écartelées entre le lointain de la globalisation et la fragmentation du national postcolonial en une mosaïque d'espaces enchevêtrés. La cité intramuros, fondée sur des liens de sang imaginaires ${ }^{11}$, lieu des origines et de la coutume, s'articule à la cité extramuros de plus en plus happée par la transnationalisation. Le politique n'étant plus un refus délibéré et conscient de la guerre, et encore moins un processus de conservation de la vie individuelle, le rapport de capture et de dépense fait fi de l'utilité de type libéral sans pour autant produire du sacré. Parce qu'elle porte directement sur la «prise du pouvoir» sans passer par la confrontation et sans déboucher sur la négociation des choix de société, l'élection prend un caractère maléfique menaçant de détruire toute possibilité de «vivre ensemble politiquement ». De la démocratie des urnes imposée/importée, on est passé à une «demon-cratie » ${ }^{12}$

10. Mbembe se trouve ici en excellente compagnie de Mamadou Diouf qui, dans le cadre des travaux pionniers du SEPHIS, a pris l'initiative d'introduire les universitaires africains aux Subaltern Studies indiennes. Provincialiser l'Occident impliquerait également ne reconnaître désormais la narration que comme un mode parmi d'autres de construction de l'identité et du rapport à l'espace-temps, voir mon échange (JewsiEWICKI 2002 : 593-599) avec MBEMBe (2002 : 629-641).

11. Ils ne le sont évidemment ni plus ni moins que ceux des nations.

12. C'est le titre d'un tableau de Chéri-Cherin, peintre urbain autodidacte de Kinshasa, R. D. Congo. 
des nations qui se sentent prises pour des lieux d'expériences politiques douteuses exposées au voyeurisme médiatique fournissant à l'Occident ses spectacles de gladiateurs. Alors, les expériences de paix et de réconciliation ne sont que des épisodes de la guerre.

Rédigé de l'intérieur de l'expérience sud-africaine, ce regard sur les sociétés au nord de Limpopo ne devrait pas être confondu avec l'afropessimisme. La lucidité et le respect des subjectivités locales sont d'indispensables sources aux solutions pragmatiques, originales et réussies à condition de ne pas ignorer le monde qui, sans être un village planétaire, ouvre un espace-temps d'interactions et d'interdépendances. La transition sud-africaine n'est en rien un miracle, mais la preuve tangible qu'aucune malédiction ne frappe l'Afrique. En octobre 1993, encore président, Frederik De Klerk déclarait à la presse : «Nous vous surprendrons tous et nous nous surprendrons peut-être nous mêmes. Ce qui va arriver à l'Afrique du Sud dans les mois et les années à venir constituera d'extraordinaires bonnes nouvelles. » À peine une décennie plus tard, au lendemain des troisièmes élections, Desmond Tutu confirmait: «Ce qui est important pour nous aujourd'hui, c'est que nous devenions une société normale ${ }^{13}$. Transformer l'extraordinaire en ordinaire, c'est ça le miracle sud-africain fascinant par la réussite de passer d'une presque certitude de guerre civile à une société de plus en plus confiante en sa normalité. En examiner les raisons n'est pas mon ambition, je souhaite seulement le rappeler afin de justifier la place accordée dans ce numéro aux débats et aux démarches de réparations et de réconciliations dans ce pays et dans l'ensemble de l'Afrique australe. La transition sud-africaine a été négociée et appliquée entre les Sud-Africains avec le présent à l'esprit, les solutions retenues ont tenu compte, sans les copier, des expériences d'Amérique latine, de Pologne, etc. Le Nord, le monde dit industrialisé, a offert son assistance non pas à titre de réparation à l'égard d'une dette, mais pour répondre à un défi du présent. Enfin, et cette opinion sera sans doute controversée, les réparations et la réconciliation ont été et demeurent un enjeu interne âprement débattu entre les Sud-Africains. L'opposition du président Thabo Mbeki aux procès engagés par certaines ONG devant les tribunaux américains pour obtenir des réparations de la part des multinationales n'est pas étrangère au souci de maintenir le flot d'investissements. De la raison première de son opposition me semble néanmoins venir la ferme conviction des hommes politiques sud-africains qu'il leur est plus avantageux de négocier avec le monde extérieur au présent et en vertu des considérations qui en relèvent.

\section{Les débats et leurs enjeux}

La composition d'un numéro spécial de revue reflète inévitablement autant le projet initial qu'elle traduit la dynamique de la recherche en cours et

13. Cités par l'Agence France-Presse, Le Soleil, 17 avril 2004, A-26. 
celle du monde où elle est engagée. Notre objet étant des plus actuels ${ }^{14}$, il n'était pas souhaitable que l'évolution du dossier ignore le contemporain et l'actuel et encore moins qu'elle se limite paresseusement à en livrer une chronique. La partie Positions, réflexions, histoire du numéro se trouve donc balisée par deux articles qui défient les évidences, celui d'Anthony Appiah et celui d'Achille Mbembe. L'honnêteté intellectuelle et le respect du droit du lecteur de disposer de l'information complète me semble exiger de faire suivre l'article d'Appiah du plaidoyer d'Ade Ajayi en tant que membre du Groupe des personnalités éminentes. Je viens d'expliquer ce choix. Suivent les analyses et les présentations critiques des cas dont le plus imposant est offert par l'Afrique du Sud.

Le Congo et la Belgique sont peut-être engagés dans une incertaine démarche de deuil, non pas encore du passé colonial mais au moins de ses mémoires. L'Afrique du Sud, où la réconciliation semble progressivement intégrée dans une démarche de reconstitution du corps de la nation qui s'est révélée à elle-même en 1994, commence à prendre sa place dans la communauté politique actuelle des peuples noirs. Les îles sont entrées de force dans le monde industriel avec la traite des esclaves africains et le sucre. S'il y a des sociétés que l'esclavage des temps modernes a façonnées en profondeur, des sociétés qui non seulement seraient différentes en son absence mais seraient radicalement autres, ce sont bien celles des Antilles et des îles de l'océan Indien. Et pourtant, gare à nous de vouloir les réduire à la traite, à l'esclavage et à leurs effets. L'engagisme (recrutement en Inde, par contrat à durée fixe, des personnes visant à fournir à l'industrie sucrière une main-d'œuvre quasi servile) vient d'abord à l'esprit pour distinguer les îles de l'océan Indien de celles des Caraïbes, puis, sur un autre plan, l'indépendance d'Haïti, etc. L'inventaire des traits distinctifs n'a pas sa place ici. Je souhaite uniquement souligner qu'il est important de présenter des études sur les expériences et les débats concrets, et d'éviter les généralisations. Le hasard plutôt que la sélection délibérée a débouché sur un équilibre des deux articles du côté de chaque océan qui borde le continent africain. La concertation avec les auteurs fournit des analyses sur ce qui aujourd'hui paraît le plus actuel : le conflit des mémoires en Haïti, qui pourrait être au cœur de la (non)-reconnaissance par l'État de sa nation; les débats aux Antilles et en Guyane françaises, mais aussi parmi les Antillais et les Guyanais vivant en France métropolitaine sur l'inégalité des chances et la discrimination raciale héritées de la traite négrière ; à la Réunion, la représentation dans l'espace public — qui équivaut à la pleine et légitime présence - de l'identité française et pas seulement citoyenne d'anciens esclaves ; enfin, à l'île Maurice un autre conflit des mémoires, une concurrence des victimes, une compétition pour l'accès à la richesse nationale

14. Au sens qu'est donné à ce terme dans le dossier, «L'actualité du contemporain », Le Genre humain, 35, Paris, Éditions du Seuil, 2000. 
entre les descendants d'esclaves noirs et ceux — aujourd'hui majoritaires d'anciens engagés indiens ${ }^{15}$.

Il aurait été inconcevable de clore ce numéro sans aborder la question de la restitution. Sans être spécifique aux contentieux qui découlent de la traite des esclaves et de la colonisation, elle en fait partie. Les restitutions d'objets considérés en Occident comme œuvres d'art et patrimoine muséal, mais néanmoins comme objets sacrés, patrimoine national, insignes de pouvoir, voire ancêtres ${ }^{16}$ dans les sociétés où ils ont été prélevés, ont fait l'objet d'ententes internationales et se trouvent en partie régies par des conventions. À partir de l'exemple de la stèle éthiopienne de Rome, Éloi Ficquet montre à quel point la restitution est problématique, même dans le cas où l'illégalité de son appropriation est évidente.

Le travail de mémoire, qu'il est impossible d'ignorer lorsque l'on traite des réparations et de la réconciliation, figure en toile de fond de plusieurs articles sans que la majorité des auteurs l'abordent explicitement. Cette question reçoit une attention explicite dans les articles qui traitent des Antilles et de l'océan Indien et dans le dossier consacré au Burundi ainsi que dans la Chronique bibliographique. La place de la mémoire et de ses enjeux dans l'espace public est frappante à la Réunion, à l'île Maurice ; elle nous touche différemment mais non moins intensément en Haïti. À l'inverse, la mémoire est écrasée au Burundi par l'Histoire chargée par des vainqueurs de parvenir à l'établissement des «vérités » définitives. Mise au silence, renvoyée dans la sphère privée, blottie au fond du religieux, la mémoire est exclue de l'espace public. Faute d'un travail de mémoire qui engage les citoyens, les institutions monopolisent les revendications de réparations et gèrent la réconciliation qui n'engagent qu'elles-mêmes, et donc personne.

15. Le sort réservé aux habitants de l'île Diego Garcia (dans l'archipel des Chagos) montre que l'abolition de l'esclavage n'a pas sonné le glas de l'imposition à des humains du statut d'êtres « jetables » (disposable people). Pour que l'île devienne exclusivement une base militaire américaine, les habitants - esclaves transportés de l'île Maurice - (LASSEMILlante 2002: 83-88), installés depuis la fin du $\mathrm{XVIII}^{\mathrm{e}}$ siècle ont dû abandonner leurs biens, leur mode de vie et l'espace-temps de leur identité. Sous prétexte de retour au lieu de départ de leurs ancêtres, plutôt lieu de leur déportation initiale comme esclaves de plantation, à la fin des années 1960, un millier de Chagossiens ont été "retournés », puis abandonnés à leur sort sur l'île Maurice. Luttant pour le droit à l'existence collective et à la dignité, ils se souviennent qu'on a eu plus d'égard pour le sort des tortues géantes qu'au leur en préférant Diego Garcia à Aldabra. Sivakumaren MARDEMOOTOO écrit : «The British therefore put a price tag of $\$ 11$ million on our people's lives and the Americans decided to give giant tortoises preferential treatment over us. When we look back at this, we tell ourselves that the British and the Americans never really realised that slavery was a wrong thing ; worst, they had not accepted that slavery had been abolished because in their mind and deeds, black people's lives could still be price tagged and were still inferior to animals. » (« The Plight of Chagossians », le 15 octobre 2001 à Port-Louis).

16. C'est le cas du corps de Saartjie Baartman dont certaines parties ont longtemps été conservées au Musée de l'Homme avant d'être récemment restituées à l'Afrique du Sud et à sa communauté ethnique. 
Omasombo et de Villers abordent la question de la dette coloniale sous ses aspects politiques et éthiques. Ils montrent aussi que les relations entre la Belgique et ses anciens territoires africains, soit à titre de possession coloniale soit à titre de mandat international, sont profondément imbriquées dans les rapports intercommunautaires en Belgique elle-même. Au-delà des mérites propres à chacun de ces articles, leur contribution au numéro vient de la fine démonstration de l'enchevêtrement «national », autant belge que congolais, que des questions qui relèvent de l'Histoire et de la mémoire du lien «colonial». Alors qu'à tort, on considère souvent pouvoir traiter la question de la «dette» politique, ethnique, matérielle de la traite des esclaves, de la colonisation comme un phénomène ne relevant que des relations interétatiques ou internationales régionales, de Villers et Omasombo montrent le contraire. La Belgique constitue à cet égard un laboratoire, sa structure communautaire mettant sans doute plus en évidence certains enjeux politiques (de Lame 2003) ${ }^{17}$. Il n'en est pas moins vrai que dette et mémoire de l'esclavage et de la colonisation ne peuvent être analysées sans tenir compte de leur actualité «nationale» de part et d'autre de l'ancien lien de dépendance. S'il y a un apport méthodologique du présent numéro aux débats sur les réparations, il se situe dans la démonstration de la profonde imbrication de ses enjeux autant dans les mémoires que dans la politique nationale de chaque pays et de chaque région concernée. L'article de Gérard Barthélémy, la belle démonstration de Françoise Vergès ou l'analyse d'Éloi Ficquet confirment l'exemplarité du cas belgo-congolais. Le Rapport Debray est explicite : «Nos rapports avec Haïti sont plus délicats, émotifs et rétractiles parce qu'en clair-obscur, ils mettent en jeu les rapports de la France avec elle-même ${ }^{18}$. Les rapports de la France avec l'Algérie, ou encore ceux avec les États africains issus de l'ancienne Afrique occidentale française sont également, et à plusieurs titres, des rapports «de la France avec elle-même ». Parce que la traite, et plus encore, la colonisation ont autant contribué à faire la France d'aujourd'hui que les sociétés qui en ont été l'objet. La colonisation a également forgé le lien en vertu duquel plusieurs sujets d'hier sont aujourd'hui ses citoyens. Ailleurs, comme en Haïti, ce passé «fait partie de notre histoire [hexagonale], mais non de notre mémoire ${ }^{19}$, c'est pourquoi l'oubli gouverne l'espace public et y empêche la circulation des mémoires privées, communautaires, etc. Ce refus d'audience opposé aux mémoires du temps de la colonisation distingue le cas français ${ }^{20}$ de celui des autres puissances occidentales qui se définissent

17. Voir la recension dans ce numéro.

18. Rapport Debray, p. 6.

19. Ibid., p. 7.

20. Le Comité indépendant de réflexion et de propositions sur les relations francohaïtiennes insiste sur la nécessité de remédier à la méconnaissance de l'Histoire et recommande « de mieux prendre en compte la colonisation et la décolonisation, et notamment les questions liées à la Traite négrière, à l'esclavage et à ses libérations, dans les diverses réécritures en cours des programmes d'histoire du collège 
aujourd'hui comme des sociétés multiculturelles. En Grande-Bretagne, non seulement Scor-Zay-Zee peut chanter dans son rap Great Britain, "C'est l'esclavage qui a fait la richesse de la Grande-Bretagne » - liberté d'expression oblige - mais cette chanson est diffusée par la $\mathrm{BBC}^{21}$.

Dans la tradition britannique, la société politique sud-africaine d'aujourd'hui affiche résolument son pragmatisme. Les jeunes Afrikaners l'opposent même aux positions de principe de leurs aînés quand ces derniers rappellent le devoir de défendre la langue, la culture, etc. (Giliomee 2003 : 664).

Pourtant, la reconnaissance de la légitimité des nouvelles identités et la reformulation du passé, tel qu'il est représenté dans l'espace public, demeurent des enjeux importants ${ }^{22}$. À l'égard de deux communautés politiques, Africains Noirs et Afrikaners (plus que d'autres Sud-Africains d'origines européennes attachés à leur africanité), l'article de Rehana Vally et celui de Bennetta Jules-Rosette et David Coplan mettent en évidence le travail de réorganisation du message que la représentation collective de soi projette dans l'espace public. Qu'il s'agisse du retour et du digne enterrement des parties du corps de Saartjie Baartman, de la commémoration de l'arrivée en 1652 des premiers colons blancs, du monument aux Voortrekkers, de «Nkosi Sikelel' iAfrika», passé de statut du chant du Congrès national africain, interdit de l'espace public sous l'apartheid, à celui d'hymne national, ou de District $\mathrm{Six}^{23}$, quartier multiculturel et multiracial du Cap rasé sous l'apartheid et représenté aujourd'hui par son musée, la réparation vise le corps mythique de la nation maltraité, mutilé, violé pendant plus de trois siècles. Au premier regard, ce sont la légitimité et la visibilité de la légitime appartenance à la nation d'une communauté politique qui sont apparentes. Plus au fond, c'est de la renaissance de la nation, de sa complétude et de l'actualité de la juste représentation de son passé dont il s'agit. Pour cette raison, le Freedom Park rassemble les monuments et les symboles anciens et nouveaux, évitant ainsi le piège du cimetière des statues désuètes ${ }^{24}$, alors que le visiteur entre dans le Musée de l'apartheid en s'identifiant, au choix, à l'expérience de «Blanc» ou de «non-Blanc» sous les lois de ce régime. Obliger le visiteur à refaire l'expérience du passé s'inspire du travail de la Commission «Vérité et réconciliation» qui voulait qu'après les audiences publiques et la publication de son rapport aucun Sud-Africain ne puisse se prévaloir de l'innocence à l'égard du passé en invoquant l'ignorance ou l'amnésie. Si la nation sud-africaine se caractérise, dès sa naissance, par la

et du lycée » (annexe D, p. 71), sans s'aventurer sur le terrain de la circulation des mémoires.

21. Libération, 5 avril 2004, p. 12.

22. Dans la Chronique bibliographique, je m'attache à montrer à quel point cette question est actuelle. Martin Botha et Samuel LeliÈvre, qui analysent dans ce numéro le film sud-africain Promised Land, dont le personnage principal cherche à renouer avec son passé, n'en disconviennent pas.

23. Le musée a obtenu, en 2003, le prestigieux Prince Claus Award.

24. C'est le cas d'un parc budapestois auquel Anne-Marie LosAnCZY (1999) consacre une passionnante analyse. 
résistance à l'oppression, son corps politique a attendu plus de trois siècles pour pouvoir vivre pleinement et ouvertement sa véritable identité. Rétablir dans l'espace public les justes représentations du passé est donc un acte de réparation et de reconnaissance. S'adressant en premier lieu à la nation ellemême, réparations et reconnaissance ont néanmoins une visée plus large dans la mesure où il s'agit également de rétablir la dignité des peuples noirs non plus dans leur gloire ancienne ou éternelle ${ }^{25}$, mais dans leur modernité et leur actualité. De la première république moderne noire en Haïti à la nation industrielle d'Afrique du Sud, au nom de la Renaissance africaine, le combat serait le même ${ }^{26}$. Pour l'homme politique qu'est Thabo Mbeki, c'est aussi l'occasion d'internationaliser et ainsi de rendre plus diffuses les tensions politiques internes autour de la « race».

Anthony Appiah et Michel Giraud soulignent que l'horreur de certains crimes contre l'humanité rend leur réparation matérielle impossible ; comment pleinement réparer les effets de l'holocauste ou de l'esclavage, surtout quand, dans leur écrasante majorité, les victimes ne sont plus ? La reconnaissance du crime et la demande de pardon, donc la repentance, semblent être les seules démarches pouvant conduire au rétablissement du lien social entre les descendants des victimes et ceux des responsables du crime. Néanmoins, si la repentance n'est pas suivie des mesures qui contribuent à rétablir la victime dans la situation politique, sociale, économique qu'elle aurait dû connaître sans le handicap causé par le préjudice subi, sa crédibilité en pâtira. Les réparations matérielles, individuelles et collectives ont été prévues en Afrique du Sud depuis la mise en place de la Commission "Vérité et réconciliation » qui devait établir la liste des bénéficiaires, fixer les montants et les modalités de versement. Warren Buford et Hugo van der Merwe présentent une analyse aussi complète que possible de l'état actuel du dossier sud-africain, puis l'étendent aux quatre autres pays de la région. C'est la première étude comparative exhaustive de la question, et nous sommes fiers de l'offrir en nouveauté au public francophone.

Les réparations contribuent à replacer les victimes et leurs descendants dans la situation qui aurait dû être la leur, mais elles n'épuisent pas toutes les mesures nécessaires pour s'assurer que le crime ne paie pas et pour empêcher sa répétition. Ceux qui l'ont commis ou commandité doivent le reconnaître, s'en repentir et être punis. La répression d'un crime de masse est difficile, parfois impossible du fait du nombre de suspects à juger et du coût économique et social du châtiment. Les emprisonner par dizaines de

25. Se distinguant ainsi de la négritude, du panafricanisme ou encore des antiquités nègres de Cheikh Anta Diop.

26. Je reviens brièvement sur cette question dans la Chronique bibliographique. Notons une reconnaissance de la légitimité politique de cette position par la Commission Debray: «Le Président Mbeki et les autorités sud-africaines [...] ont déjà manifesté un vif engagement personnel envers un pays-symbole à leurs yeux », Rapport Debray, p. 52. 
milliers comporte le risque que les victimes et leurs descendants, en supportant les coûts, s'en trouvent davantage appauvris. Si le but est d'aboutir à la reconstruction du lien social endommagé ou détruit, une répression en grand nombre peut être jugée contre-productive. Pourtant, l'impunité ou l'amnistie générale et inconditionnelle sont inacceptables. En Afrique du Sud, une solution originale a été trouvée par le législateur et mise en œuvre par la Commission «Vérité et réconciliation ». L'amnistie des crimes commis pour des motifs politiques a été accordée en échange d'aveux pleins et complets suivis, en principe, d'un repentir public. Graeme Simpson, dans le cadre des activités du Center for the Study of Violence and Reconciliation qu'il dirige, suit ces questions et en donne une lecture lucide et avertie. Le crime accouche d'un autre crime (« un serpent fait naître un serpent», dit le proverbe zoulou qu'il cite). Néanmoins, l'amnistie peut-elle rompre le cercle de la violence ou au contraire risque-t-elle de l'encourager, voire de le généraliser ? Faut-il adapter la justice au crime, appliquer rétroactivement des normes particulières à la période de transition? L'amnistie exclut le crime motivé par la haine raciale mais, sous l'apartheid, la race dominait la politique. Est-il alors juste de l'en exclure a posteriori $?^{27}$ La frontière entre la politique et le crime se trouve donc remise en question alors que la majorité des citoyens ne souhaitent pas qu'elle s'efface. Comment rétablir le respect du citoyen pour la loi et sa confiance en la réconciliation alors que les mesures de réparation paraissent moins généreuses que l'amnistie envers des criminels protégés contre les poursuites, même civiles ?

Appiah remarque que la réparation matérielle échappe à notre maîtrise au fur et à mesure que l'on s'éloigne du temps du crime, même imprescriptible. Il ne resterait que la réparation expressive, mais elle non plus n'est pas nécessairement recevable pour les descendants des victimes. L'esclave ayant été, avant l'abolition de l'esclavage, reconnu par la loi comme un bien plutôt que comme un être humain, cette déshumanisation allait stigmatiser les anciens esclaves pour longtemps. Gérard Bathélémy écrit que «toute indemnité ne peut être perçue par l'ancien esclave que comme une tentative officielle pour confirmer rétroactivement le droit sur lui de son ancien maître ». Voici le point névralgique de collision entre les mémoires familiales et les rituels officiels de commémoration des luttes contre l'esclavage et de ses diverses abolitions, entre les demandes de réparation de nature institutionnelle et les attentes individuelles de la part des descendants d'esclaves. Même en Haïti, pays fier d'avoir arraché sa liberté par les armes, l'esclavage d'ascendants est couvert par l'amnésie. Les recherches de Sudel

27. Il n'est pas besoin d'être un fin connaisseur de la situation sud-africaine pour comprendre qu'aussi surprenante que puisse paraître l'exclusion du principe de l'apartheid de la qualification de politique, il aurait été impossible de faire autrement sans absoudre le régime de l'apartheid de ce qui l'a contraint à se saborder, la condamnation politique et morale pour le crime politique. Peu avant sa fin, même l'église réformée de Hollande (Nederduits Gerreformeerde Kerk) a pris ses distances (GILIOMEE 2003 : 620-622). 
Fuma à la Réunion attestent également de la réticence à se souvenir de l'ascendance des esclaves ${ }^{28}$. La reconnaissance de l'esclavage comme crime, l'espoir de bénéficier d'une réparation, la discrimination positive à l'égard des descendants d'anciens esclaves, encore qu'elle les pousse à se définir comme communauté de race $^{29}$, contribuent, depuis peu, au mouvement d'anamnèse. L'île Maurice, qui fait l'objet de l'article de Jocelyn Chan $\mathrm{Low}^{30}$, se trouve à cet égard dans une situation particulière.

Au sud du Sahara, y compris en Afrique du Sud (Ward \& Worden 1998), la mémoire de la traite des esclaves et de ses abolitions est controversée à partir du moment où l'on s'écarte des forts d'où partaient les esclaves déjà aux mains des négriers occidentaux. Dans tous ces pays, le souvenir de la capture en esclavage à la suite des guerres et des razzias entre les royaumes, les chefferies et les ethnies est encore vif. À l'est de l'Afrique et à Madagascar, à peine trois générations séparent ces événements du présent. Partout l'esclavage domestique est d'une actualité brûlante et récurrente (Journal des africanistes 2001). Les abolitions ont été imposées de l'extérieur et de surcroît ont légitimé l'imposition du règne colonial alors que la colonisation a été vécue comme forme d'esclavage, en particulier pour les chrétiens qui se la représentaient comme l'esclavage d'Égypte attendant la venue d'un Moïse. Les commémorations officielles répondent donc plus à une demande internationale qu'à un besoin local, elles se conforment plutôt au rituel diplomatique qu'elles n'ouvrent l'espace public aux mémoires.

En Occident, en dehors des États-Unis, l'histoire de la traite des esclaves, peu connue du public, ne rencontre aucune mémoire, ou presque. Là où elle apparaît, c'est grâce aux citoyens originaires de l'immigration. Le rapport de la Commission Debray insiste sur la nécessité d'offrir une plus grande place à l'histoire de la traite dans l'enseignement. Après une longue période d'amnésie, l'histoire de la colonisation fait son entrée dans les manuels français, en suivant plutôt qu'en précédant l'histoire de l'immigration. Depuis une dizaine d'années, quelques groupes et diverses personnes, surtout l'ACHAC (Association connaissance de l'histoire de l'Afrique contemporaine), mènent avec succès, courage et persévérance un combat pour une meilleure connaissance de cette période. Il a cependant fallu que paraissent Le passé d'une illusion de François Furet (1995), puis Le livre noir du communisme (Courtois et al. 1997), pour que s'engage un vrai débat sur la guerre d'Algérie faisant place aux mémoires pour que le public français soit prêt au procès

28. Communication personnelle dont je remercie l'auteur.

29. Dans un excellent article, Anne-Marie LosANCZY (à paraître) montre comment les mesures de réparation contribuent à communautariser les citoyens colombiens d'origine africaine et à effacer la distinction, jadis forte, entre les descendants des marrons et ceux des esclaves libérés.

30. En complément de son article dans ce numéro, il est utile de consulter son ouvrage collectif (CANGY, Chan Low \& PAROOMAL 2002 : 83-88). Ces actes du colloque, tenu sous l'égide de l'UNESCO en 1998, présentent une bonne image de la diversité d'opinions et d'intérêts. 
de la colonisation. Le livre noir du colonialisme (Ferro 2003) complète le procès $\mathrm{du} \mathrm{Xx}^{\mathrm{e}}$ siècle occidental, de ses mémoires et de ses crimes. Depuis Hannah Arendt, il nous faut reconnaître le fascisme, le communisme et le colonialisme comme étant trois lieux de mémoire de l'Occident contemporain. La réflexion à deux voix que Jane Burbank et Frederick Cooper présentent au sujet du Livre noir du colonialisme (voir Analyses et comptes rendus dans ce numéro), replacé dans la perspective du Livre noir du communisme, élargit le cadre du questionnement sur les responsabilités et les réparations. Bennetta Jules-Rosette et Rehana Vally la prolongent.

L'année 2004 est riche en bicentenaires d'événements. Comment se soustraire au rituel contemporain de la commémoration ? Cette année a été proclamée l'année du bicentenaire de l'abolition de l'esclavage. Le directeur général de l'UNESCO visite les hauts lieux de la route des esclaves d'où il fait des proclamations régionales. C'est aussi le bicentenaire de l'indépendance d'Haït, première république noire indépendante, moderne, contemporaine des révolutions américaine et française. Même si son indépendance a été éphémère, Haïti a été le premier pays à en tester la portée universelle. C'est enfin le dixième anniversaire des premières élections libres et multiraciales en Afrique du Sud. De ces trois événements historiques, seul le dernier peut, sans hésitation, être qualifié de success story. La nouvelle Afrique du Sud se réclame de la continuité tant historique que mémorielle des luttes pour son émancipation et celle des peuples noirs ${ }^{31}$. Entre la révolution haïtienne qui a proclamé tous ses citoyens noirs, donc égaux quelle que soit la couleur de leur peau, et l'Afrique du Sud de Thabo Mbeki qui a déclaré tous les Sud-Africains africains quelle que soit leur communauté d'appartenance (de race), sont nés et morts le colonialisme et l'apartheid. Aucune de ces déclarations n'a malheureusement pu devenir un principe d'action politique et encore moins une règle de vie sociale; en Haïti comme en Afrique du Sud la race demeure au cœur du politique et reste un facteur d'inégalité.

Université Laval, Québec.

31. J'en parle dans la Chronique bibliographique. Dans son ouvrage, Stephen SмIтH (2003 : 108) remarque très justement que pour avoir été «basculés dans un monde qu'ils ne reconnaissent pas comme le leur », les Africains considèrent l'effacement de ce «viol d'identité » comme une importante mesure de réparation. Habiter le monde moderne à sa manière est un redoutable défi auquel sont confrontées de nombreuses sociétés ayant accédé au monde postmoderne via la traite des esclaves et la colonisation. La tentation reste grande d'approprier les modes de construction de soi de l'ancien maître plutôt que de construire les siens, voir Achille Mbembe (2000) et mon commentaire (JEwsIEwICKI 2002) dans le numéro de Public culture, op. cit. 


\section{BIBLIOGRAPHIE}

BAKO ARIFARI, N.

2001 «La mémoire de la traite négrière dans le débat politique au Bénin dans les années $1990 »$, Journal des africanistes, 70 (1-2) : 221-232.

BERLIN, I.

2004 Generations of Captivity : A History of African American Slaves, Cambridge, Balknap Press/Harvard University Press.

BotTe, R.

2001 «De l'esclavage et du daltonisme dans les sciences sociales. Avant-propos », Journal des africanistes, 70 (1-2) : 7-42.

BotTe, R. (dir.)

2001 «L'ombre portée de l'esclavage. Avatars contemporains de l'oppression sociale », Journal des africanistes, 70 (1-2).

Cangy, J.-C., Chan Low, L. J. \& Paroomal, M.

2002 L'esclavage et ses séquelles: Mémoire et vécu d'hier et d'aujourd'hui, Réduit, Presses de l'Université de Maurice.

Courtois, S. et al.

1997 Le livre noir du communisme: crimes, terreur, répressions, Paris, Robert Laffont.

FERRO, M. (dir.)

2003 Le livre noir du colonialisme. $X V I^{e}-X X I^{e}$ siècle : de l'extermination à la repentance, Paris, Robert Laffont.

FREDRICKSON, G.

2004 «America's Original Sin», New York Review of Books, 25 mars.

FURET, F.

1995 Le passé d'une illusion : Essai sur l'idée du communisme au XXe siècle, Paris, Robert Laffont.

GiLIOMEe, H.

2003 The Africaners. Biography of a People, London, Hurst \& Co.

HARTOG, F.

2003 Régimes d'historicité. Présentisme et expérience du temps, Paris, Éditions du Seuil.

JEWSIEWICKI, B.

2002 The Subject in Africa: In Foucault's Footstepts, Public Culture, 14 (3) : 593-599. 
JOURNAL DES AFRICANISTES

2001 «L'ombre portée de l'esclavage. Avatars contemporains de l'oppression sociale », numéro spécial, 70 (1-2).

DE LAME, D.

2003 «Deuil, commémoration, justice dans les contextes rwandais et belge », Politique africaine, $92: 39-55$.

LASSEMillante, $\mathrm{H}$.

2002 «L'esclavage aux Chagos pendant les dix-huitième et dix-neuvième siècles », in J.-C. CAngy, J. Chan Low \& M. Paroomal, L'esclavage et ses séquelles: Mémoire et vécu d'hier et d'aujourd'hui, Réduit, Presses de l'Université de Maurice : 83-88.

LosAnCZY, A.-M.

1999 «Le patrimoine de l'oubli. Le "Parc-musée des statues" de Budapest», Ethnologie française, 29 : 445-452.

À paraître " "Sentirse negro". Des empreints du passé à la mémoire collective parmi les groupes noirs de Colombie », Annales. Histoire. Sciences sociales.

Mam Lam Fouck, S.

2001 «Les sociétés créoles des départements français d'Amérique et le fait esclavagiste : une laborieuse reconnaissance », Journal des africanistes, 70 (1-2) : 145-171.

Mbembe, A.

2000 «À propos des écritures africaines de soi », Politique africaine, 77 : 16-43.

2002 «On the Power the False», Public culture, 14 (3) : 629-641.

Robinson, R. N.

1999 The Debt: What America Owes to Blacks, London, Routledge.

Sмiтн, S.

2003 Le fleuve Congo, Arles, Actes Sud.

WARD, K. \& Worden, K.

1998 «Commemorating, Suppressing and Invoking Slavery», in S. NutTaL \& C. Coetzee, Negotiating the Past: The Making of Memory in South Africa, Cape Town : 201-217. 